\title{
Laboratory-based x-ray phase contrast CT technology for clinical intra-operative specimen imaging
}

Lorenzo Massimi, Charlotte K. Hagen, Marco Endrizzi, Peter R. T. Munro, Glafkos Havariyoun, et al.

Lorenzo Massimi, Charlotte K. Hagen, Marco Endrizzi, Peter R. T. Munro, Glafkos Havariyoun, P. M. Sam Hawker, Bennie Smit, Alberto Astolfo, Oliver J. Larkin, Richard M. Waltham, Zoheb Shah, Stephen W. Duffy, Rachel L. Nelan, Anthony Peel, Tamara Suaris, J. Louise Jones, lan G. Haig, Alessandro Olivo, "Laboratory-based x-ray phase contrast CT technology for clinical intraoperative specimen imaging," Proc. SPIE 10948, Medical Imaging 2019: Physics of Medical Imaging, 109481R (1 March 2019); doi: 10.1117/12.2511770 


\title{
Laboratory-based X-ray Phase Contrast CT technology for clinical intra-operative specimen imaging
}

\author{
Lorenzo Massimi $^{\mathrm{a}}$, Charlotte K.Hagen ${ }^{\mathrm{a}}$, Marco Endrizzi ${ }^{\mathrm{a}}$, Peter R.T.Munro ${ }^{\mathrm{a}}$, Glafkos \\ Havariyoun $^{\mathrm{a}}$, P. M. Sam Hawker ${ }^{\mathrm{b}}$, Bennie Smit ${ }^{\mathrm{b}}$, Alberto Astolfo ${ }^{\mathrm{b}}$, Oliver J. Larkin ${ }^{\mathrm{b}}$, Richard \\ M.Waltham ${ }^{\mathrm{b}}$, Zoheb Shah ${ }^{\mathrm{c}}$, Stephen W.Duffy ${ }^{\mathrm{c}}$, Rachel L.Nelan ${ }^{\mathrm{c}}$, Anthony Peel ${ }^{\mathrm{d}}$, Tamara \\ Suaris $^{\mathrm{d}}$, J.Louise Jones ${ }^{\mathrm{c}, \mathrm{d}}$, Ian G.Haig ${ }^{\mathrm{b}}$, and Alessandro Olivo ${ }^{\mathrm{c}}$ \\ ${ }^{a}$ Department of Medical Physics and Biomedical Engineering, University College London, \\ Gower St, London WC1E 6BT, UK \\ ${ }^{b}$ Nikon, X-Tek Systems Ltd., Tring Business Centre, Icknield Way, Tring, Hertfordshire, HP23 \\ 4JX, UK \\ ${ }^{\mathrm{c}}$ Barts and the London School of Medicine and Dentistry, Queen Mary University of London, \\ Newark St, London E1 2AT, UK \\ ${ }^{d}$ St. Bartholomews Hospital, Barts Health NHS Trust, West Smithfields, London EC1A 7BE, \\ UK
}

\begin{abstract}
The design of an X-ray phase contrast tomography system for intra-operative specimen imaging based on edge illumination is presented. The use of edge illumination makes possible working with large focus, polychromatic $\mathrm{X}$-ray sources reducing acquisition times of tomography scans down to values compatible with clinical use, while maintaining phase sensitivity in a compact device. The results collected so far show that application of this technology to breast conservation surgery has great potential to reduce re-operations, thus saving additional costs for healthcare services and stress for patients.
\end{abstract}

Keywords: Phase contrast imaging, edge illumination, breast tumour, intra-operative imaging

\section{INTRODUCTION}

Applications of X-ray Phase Contrast imaging (XPCi) are rapidly expanding, thanks to its ability to visualize and quantify features invisible to conventional X-ray imaging techniques on scales spanning from tens of microns to nanometers. ${ }^{1-5}$ While for a long period this technique was limited to synchrotron radiation facilities, the development of new phase detection methodologies now enables XPCi with laboratory-based sources. ${ }^{6-10}$

Despite XPCi not yet being available in hospitals, the improved visualization capabilities demonstrated so far suggest that healthcare would strongly benefit from a widespread application of XPCi. ${ }^{11-15}$ While in-vivo applications based on conventional sources are still not possible, the availability of a lab-based XPCi technology may have a significant impact in all medical practices where imaging of excised tissue is required. In particular, we targeted breast conservation surgery, where the ability to detect tumour presence in the margins of the resected tissue is essential. ${ }^{16,17}$ Most breast operations these days are based on a conservative approach, which aims at removing the cancerous tissue plus a safety margin instead of the entire breast. ${ }^{18}$ This approach has evident advantages for the patient, from faster recovery times to reduced psychological stress. However, the associated risk is the incomplete removal of the tumour, leading to recurrences and new operations with a high cost for the healthcare service and stress for the patient. Assessment of tumour margins is currently achieved through histological sectioning, with processing time of the order of days/weeks, especially for large excisions, which also delays adjuvant therapies. Thanks to its higher inherent contrast compared to standard attenuation tomography, XPCi is an ideal candidate to fulfil this unmet medical need. However, the implementation of phase

Further author information: (Send correspondence to Lorenzo Massimi)

E-mail: 1.massimi@ucl.ac.uk

Medical Imaging 2019: Physics of Medical Imaging, edited by Taly Gilat Schmidt, Guang-Hong Chen, Hilde Bosmans, Proc. of SPIE Vol. 10948, 109481R · @ 2019 SPIE · CCC code: 1605-7422/19/\$18 · doi: 10.1117/12.2511770 
contrast in a tomography scanner compatible with intra-operative imaging presents several challenges in the scanning time and machine design.

We present a tomographic scanner based on edge-illumination XPCi specifically designed for intra-operative breast imaging. The use of polychromatic X-ray sources coupled with a set of masks provides quantitative Xray phase sensitivity in a compact device. The results obtained so far with a laboratory prototype of such a scanner demonstrate that the combination of volumetric information provided by tomography and boost in image contrast by phase sensitivity delivers an outstanding amount of diagnostic information invisible to conventional systems used clinically, in a time compatible with intra-operative use. Finally, a scale-up design is also discussed in order to better fulfill clinical requirements in terms of specimen size.

\section{METHODS}

\subsection{Edge illumination}

Edge illumination (EI) is an incoherent and achromatic phase imaging technique. It holds great promise for translation into mainstream applications due to its capability to work with conventional X-ray sources. ${ }^{7,19,20}$ The working principle of EI is schematically shown in Fig.1a and b. An X-ray beam is split into a series of

(a)

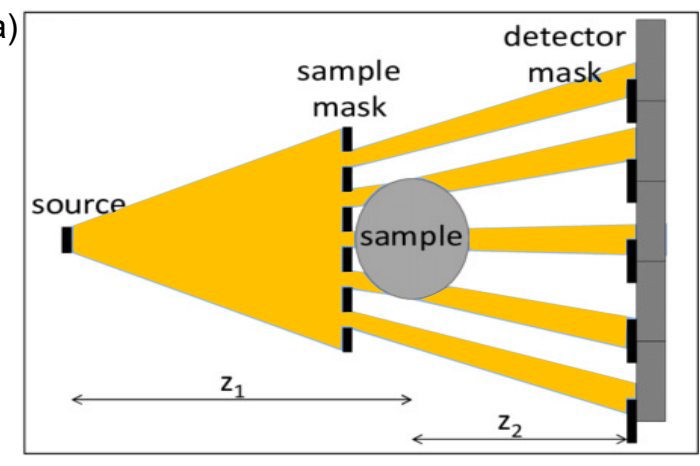

(b)

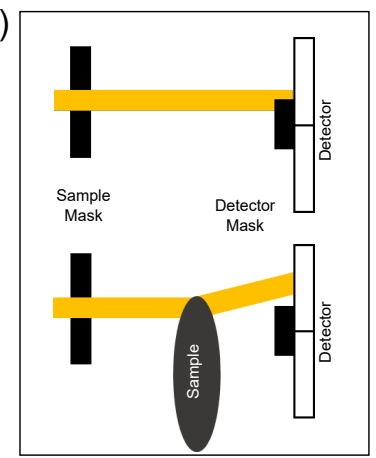

(c)

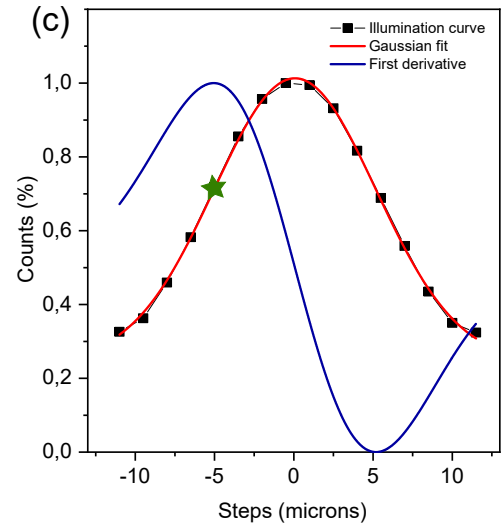

Figure 1. Panel (a) shows a scheme of an edge illumination setup based on a conventional divergent X-ray source. Panel (b) shows the working principle of edge illumination. Panel (c) shows an example of an illumination curve obtained with the edge illumination setup presented in this manuscript. The black squares are experimental data, the red curve is a Gaussian fit. The blue line represents the first derivative of the Gaussian fit.

beamlets by using a mask alternating X-ray transparent apertures and absorbing septa placed in front of the sample (sample mask). The generated beamlets will propagate to the detector where a second mask (detector mask) is placed, see scheme in Fig.1a. The system is aligned in such a way that part of the beamlets fall into the pixel and the remaining part is absorbed by one of the septa (see Fig.1b). This condition establishes a ground level of illumination in each pixel, dependent on the relative displacement between sample mask and detector mask. The change in transmitted intensity through the mask as a function of their relative position (usually the detector mask is fixed and sample mask is moved) defines the so called illumination curve, which goes from a maximum when masks are in phase to a minimum when they are out of phase. An example of an illumination curve obtained with the setup presented in this manuscript is shown in Fig.1c). When a sample is inserted in the beam path the refraction causes a shift of beamlets towards or away from the pixel, leading to a change of the intensity detected by the pixel which depends on the refraction angle (see Fig.1c). The relationship between beamlet shift and change of intensity in a pixel is described by the illumination curve, since a beamlet shift is equivalent to a displacement of the sample mask from the detector point of view. Maximum refraction sensitivity is achieved when the displacement of the sample mask corresponds to the maximum slope position on the illumination curve, since in this case a change in beamlet position is translated to the highest intensity variation measured by the pixel. The first derivative of the measured illumination curve obtained with the presented system is shown in Fig.1c where the green star marks the maximum slope position. It is worth 
noting that the use of straight linear apertures limits phase sensitivity to a single direction i.e. orthogonal to apertures. However, 2D apertures have been proposed to recover phase sensitivity in both directions. ${ }^{21}$

The illumination curve is a property of an edge illumination setup defined by the source size and shape and by the mask design. It reflects the refraction sensitivity of the system and can be mathematically described as:

$$
I C(x)=\left(A_{1} * S * A_{2}\right)(x)
$$

where $*$ denotes convolution at the detector position, $x$ is the relative displacement between sample and detector mask. $A_{1}$ and $A_{2}$ are periodic box functions describing the masks, both projected onto the detector plane to account for system magnification. $\mathrm{S}$ is the source size projected on the detector given by $\mathrm{d}=\mathrm{D}(\mathrm{M}-1)$, where $\mathrm{D}$ is the real source size and $\mathrm{M}$ is the magnification at the sample mask position. Finally, it is worth noting that while edge illumination strongly relaxes the constraints on the source size, this has to be small enough to prevent overlap of adjacent beamlets. However, the choice of system magnification allows to adjust the projected source size making the use of non-microfocus sources possible.

Images acquired with edge illumination contain a mix of information related to the attenuation and refraction properties of the sample. ${ }^{9}$ In addition, a small angle scattering signal, usually called dark-field, is also present, which relates to the sub-pixel structure of the investigated sample. ${ }^{22,23}$ An edge illumination image (for each beamlet) can be described as:

$$
\frac{I(x)}{I_{0}}=T(I C * S)(x-\Delta x)
$$

where $\mathrm{T}$ is the percentage of transmitted beam, IC is the illumination curve, $\mathrm{S}$ is the scattering function describing the illumination curve broadening due to small angle scattering. ${ }^{9,24}$ The convolution between IC and S appears shifted by $\Delta x$ due to refraction, where $\mathrm{x}$ is the coordinate in the direction normal to the aperture. ${ }^{9} \Delta x$ is related to the refraction angle $\theta_{R}$, and thus to the phase shift caused by the sample. Specifically:

$$
\theta_{R}=\Delta x / z_{o d}=\frac{d \Phi}{d x}=\frac{d}{d x} \int \delta(x, y, z) d z
$$

where $\mathrm{k}$ is the wave vector, $z_{\text {od }}$ is the sample - detector distance, and $\delta$ is the unit decrement of the real part of the sample's refractive index and $\mathrm{z}$ is the coordinate along the beam propagation direction. Likewise, $\mathrm{T}$ can be easily related to the integrated attenuation coefficient $\beta$ :

$$
T=e^{-\int \beta(x, y, z) d z}
$$

The separate extraction of maps for all the processes contributing to image formation is called phase retrieval. Under the assumption of negligible scattering, at least two images are required to retrieve maps of phase and attenuation coefficients. ${ }^{25}$ If scattering retrieval is also required, at least three images are needed. ${ }^{9}$ Finally, phase retrieval can be extended to tomography by application of the retrieval algorithm to all projections, followed by creation of sinogram for each extracted signal. Therefore, distribution of phase and attenuation coefficients for each voxel in the sample can be reconstructed. ${ }^{26,27}$ This is strictly valid in the monochromatic case, while with polycromatic beams a weighted average value over the spectrum is obtained. ${ }^{28}$ However, in the intra-operative case where short scanning time is the main requirement, the retrieval of all information is not feasible, due to the long scanning required by the acquisition of at least three images for each projection angle. To overcome this limitation, phase retrieval approaches based on the acquisition of a single image have been developed. ${ }^{29,30}$ The acquisition of a single image allows to continuously rotate the sample, thus decreasing scanning time and making tomography compatible with the requirements of intra-operative imaging.

\subsection{Experimental setup}

The experimental setup currently under test and which is expected to be used as the basis for the development of a commercial scanner is shown in Fig.2a. The source to detector distance is $85 \mathrm{~cm}$, while the source to sample distance is $70 \mathrm{~cm}$, giving a geometrical magnification of about 1.25 . The sample stage is placed as close as possible to the sample mask, as is the detector mask to the detector, leaving however a distance of few centimeters which is required in both cases to accommodate motors stages. 

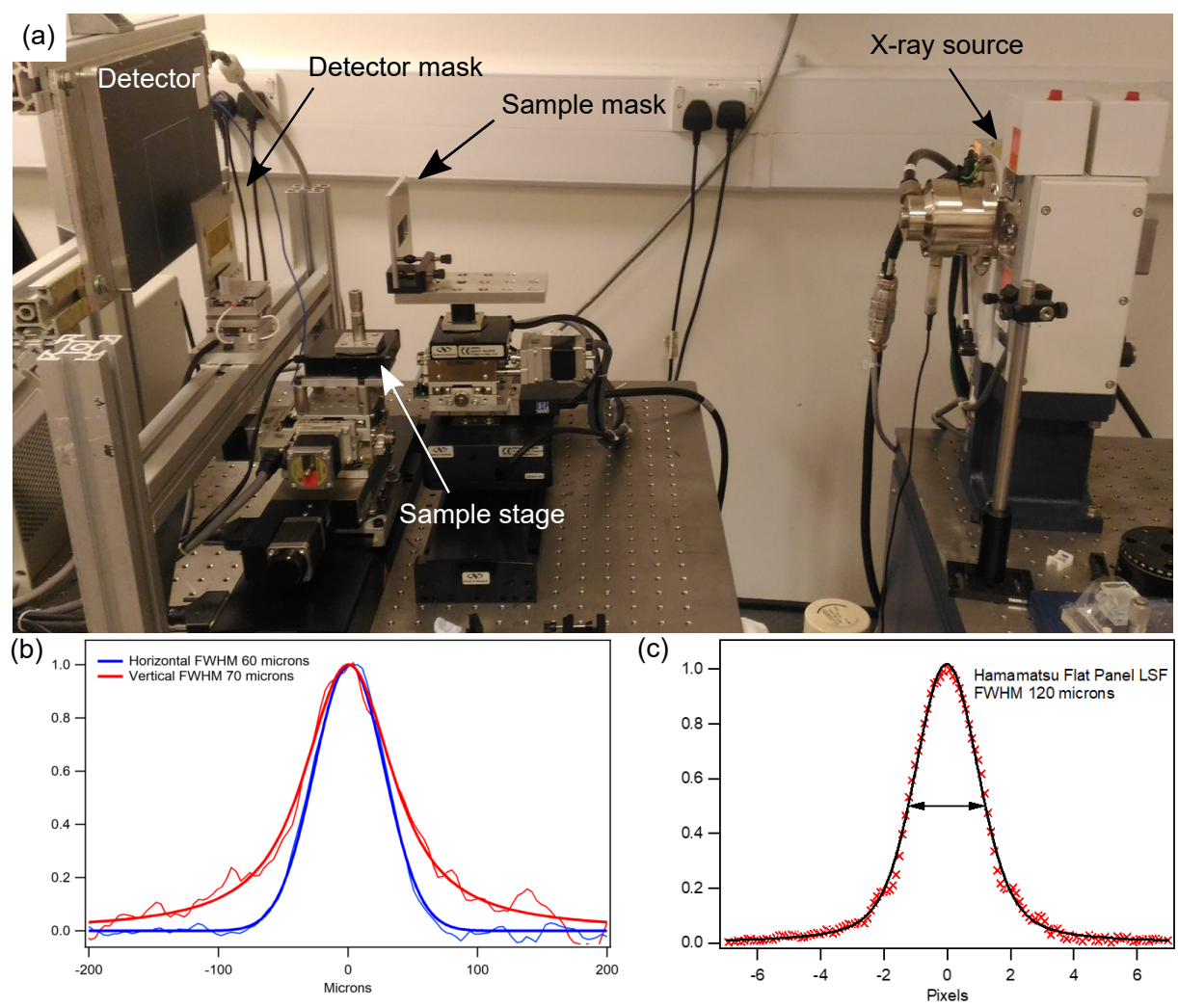

Figure 2. Panel (a) shows a picture of the phase contrast edge illumination setup currently in use at University College London (UCL). Panel (b) shows a Gaussian and Lorentzian fit superimposed to the experimental data for the source size in horizontal and vertical directions, respectively. Panel (c) reports the line spread function of the detector along the horizontal direction. Graphs in panels (a) and (b) are normalized to the maximum.

The source is a Rigaku MicroMax 007 equipped with a rotating Mo anode, operated at $40 \mathrm{kVp}$ and $20 \mathrm{~mA}$. The source has been characterized in terms of spot size, with results shown in Fig.2b. A remarkable difference in the vertical (parallel to the mask apertures) and horizontal (orthogonal to the mask apertures) direction is found. In the horizontal direction, the source is Gaussian shaped with a full width at half maximum (FWHM) of $70 \mu \mathrm{m}$ as shown by the Gaussian fit reported in Fig.3b (blue curve). In the vertical direction a similar FWHM is observed, but with longer tails compatible with a Lorentzian profile (see red curve in Fig.2b). It is worth noting that long tails in the vertical direction do not affect the shape of the illumination curve and therefore the phase sensitivity, since this applies only to the horizontal direction i.e. the one orthogonal to the apertures. Moreover, taking geometric magnification into account, the projected source size at the detector plane is below $20 \mu \mathrm{m}$ FWHM in both directions.

The detector is the Hamamatsu C9732DK flat panel with pixel size of $50 \times 50 \mu m^{2}$ and an overall active area of $120 \times 120 \mathrm{~mm}^{2}$. Its line spread function has been measured by the slanted edge method, and is shown in Fig.2c. ${ }^{31}$ The fit shows a good agreement with a Gaussian shape. A FWHM of $120 \mu \mathrm{m}$ (approximately two pixels) is found. Thus, a non negligible signal is detected by the second neighbours while a single pixel (or column) is illuminated. This cross-talk is mainly due to light diffusion in the scintillator layer, limiting the achievable resolution. ${ }^{32,33}$ No difference is observed between line spread functions in the horizontal and vertical directions.

Sample and detector masks are manufactured by gold electroplating a patterned graphite substrate, resulting in a periodic array of absorbing (gold) and transmitting (graphite) columns. A view of the mask at different scales is shown in Fig.3a and b. The graphite substrate thickness is $400 \mu \mathrm{m}$ while gold in the absorbing septa is thicker than $120 \mu \mathrm{m}$. This value ensures a negligible transmission through the septa. In addition, gold columns 
(a)

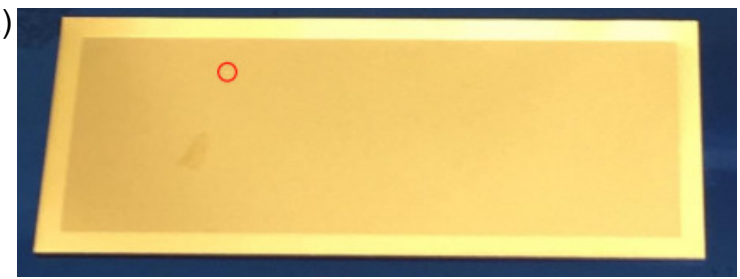

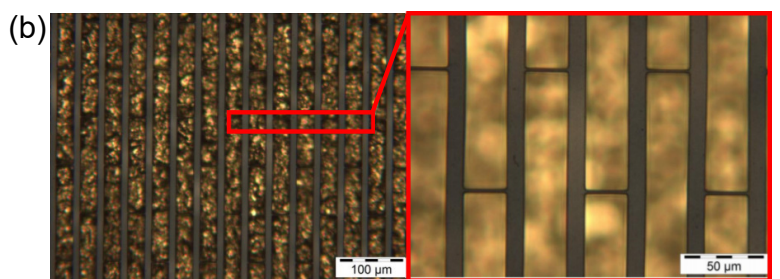

Figure 3. Panel (a) shows a view of the masks in use in the laboratory prototype. Panels (b) shows a magnified detail of the same mask. The periodic array of gold absorbing septa and graphite apertures is seen.

are not continuous but formed by different stacked columns separated by horizontal graphite bridges to provide a better stability of the layer itself (see inset in Fig.3b). The pitch and aperture size are $12 \mu \mathrm{m}(20 \mu \mathrm{m})$ and $38 \mu \mathrm{m}(48 \mu \mathrm{m})$ respectively for the sample (detector) mask. Both pitch and aperture size of the sample mask projected on the detector plane approximately match the corresponding sizes of the detector mask. The area is $55 \times 20 \mathrm{~mm}^{2}$ for the sample mask and $60 \times 25 \mathrm{~mm}^{2}$ for the detector mask.

In terms of acquisition time this system is capable of delivering $2 \mathrm{D}$ phase contrast radiography in a few seconds, matching the performances of the currently used intra-operative radiography machines. In addition, our system delivers a full tomographic scan in less than 10 minutes for samples about 30 mm thick. The achieved scanning time fulfils intra-operative requirements allowing the surgeon to get a tomography scan while the patient is still under anaesthesia. In general, the contrast to noise ratio depends on the sample thickness, which may require an increase in scanning time for larger samples.

\subsection{Scale up design}

While the system described so far is compatible in terms of scanning time with intra-operative use, a scale up in size is required to better match the clinical needs. An analysis of the distribution of the sizes of wide local excisions (WLEs) observed at St. Bartholomews Hospital (London) is reported in Fig.4a alongside the corresponding noise performance of the proposed EI setup (Fig.4b). The distribution of the sizes of WLEs is an important factor in selecting the field of view (FoV) specification of the scanner. The graph shows the percentage
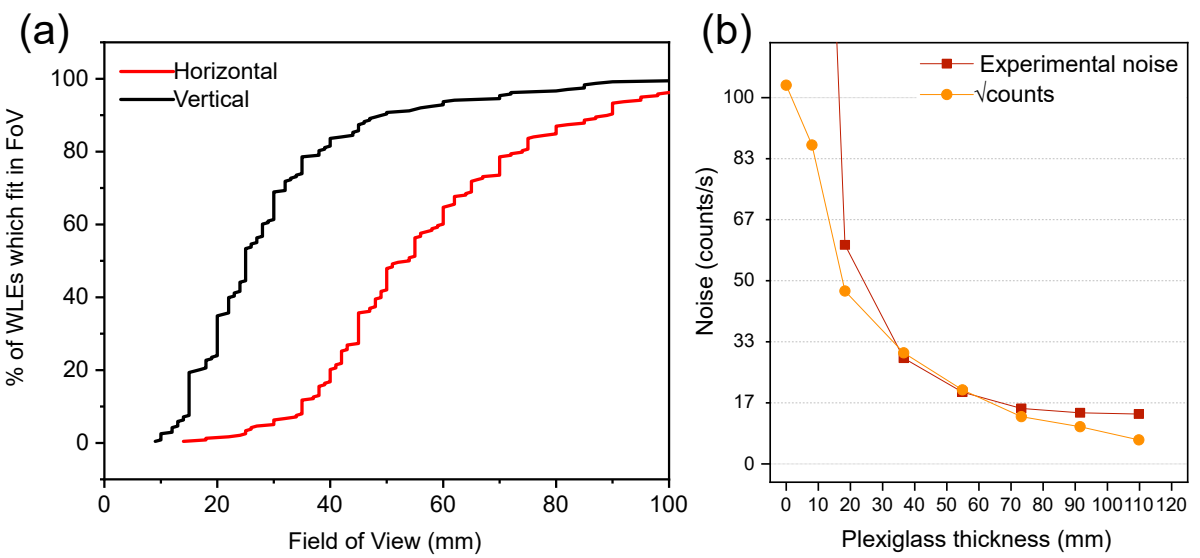

Figure 4. Panel (a) reports the percentage of WLEs which fit the field of view of the proposed EI scanner as function of FoV in both the horizontal and vertical directions. The vertical direction is defined as the direction of anterior-posterior axis. The posterior direction (for WLEs) is inwards towards the spine. Therefore, the horizontal FoV is the maximum between medial-lateral and superior-inferior axes. Superior is towards the head and inferior is towards the feet. Medial is towards the sternum, lateral is towards the nearest shoulder. Panel (b) shows the experimental noise as function of plexiglas (used as a proxy for breast tissue) thickness. The theoretical value for noise under the hypothesis of Poisson's distribution is also shown (square root of number of counts).

of WLEs extracted from a set of 248 WLEs which can be scanned within a given FoV. In the vertical direction, 
an $80 \mathrm{~mm}$ FoV covers about $96 \%$ of WLEs, and it would reach $100 \%$ if the FoV were to be increased to $100 \mathrm{~mm}$. In the horizontal direction, an $80 \mathrm{~mm}$ field of view covers about $87 \%$ of the WLEs, and achieving $98 \%$ coverage would require an increase to $136 \mathrm{~mm}$ (60\% larger).

To show the impact this would have on our scanner, the experimental noise measured as the standard deviation of the image for different sample thicknesses is shown in Fig.4b. The theoretical noise value under assumption of Poissonian distribution, i.e. the square root of number of counts, is also shown. A large deviation between theoretical and the experimental values is observed for low sample thicknesses. This is explained by the different energy response of the detector to a polychromatic beam. Increasing the sample thickness results in more beam hardening, with a consequently reduced energy spread (see Fig.6b), and thus a response of the detector closer to a value proportional to the number of photons. Finally, a large deviation is also observed around $80 \mathrm{~mm}$ of sample thickness where the experimental noise becomes constant, reaching the detector ground noise. This is the maximum sample size that can imaged with the proposed EI system. It represents a good compromise, covering approximately $96 \%$ of clinical WLEs.

Masks have proved to be the most critical component of the system to scale up. While the masks currently in use in the laboratory prototype cover a small portion of the detector, a scaled up design will use masks up to $12 \mathrm{~cm}$, fully exploiting the size of the detector. Increase in mask size poses constraints on mask flatness and stiffness. The first prototypes of masks revealed that some bending may occur. Bending affects the system performance as it may change the mask period locally. This will move the working point on the illumination curve across the field of view, changing the phase sensitivity. Moreover, the combination of larger size and thin graphite substrate creates some concerns also about the mask's mechanical rigidity. In order to overcome these problems the masks designed for the scaled up system include thicker $(1 \mathrm{~mm})$ graphite substrate. This adds $1 \mathrm{~mm}$ more of graphite in total on the beam path with respect to the setup currently in use. The effect of the increased graphite thickness on beam transmission and shape is shown in Fig.5a for different sample sizes. Plexiglas has been used to simulate sample attenuation thanks to its attenuation coefficient $\left(0.84 \mathrm{~cm}^{-1}\right.$ at 18
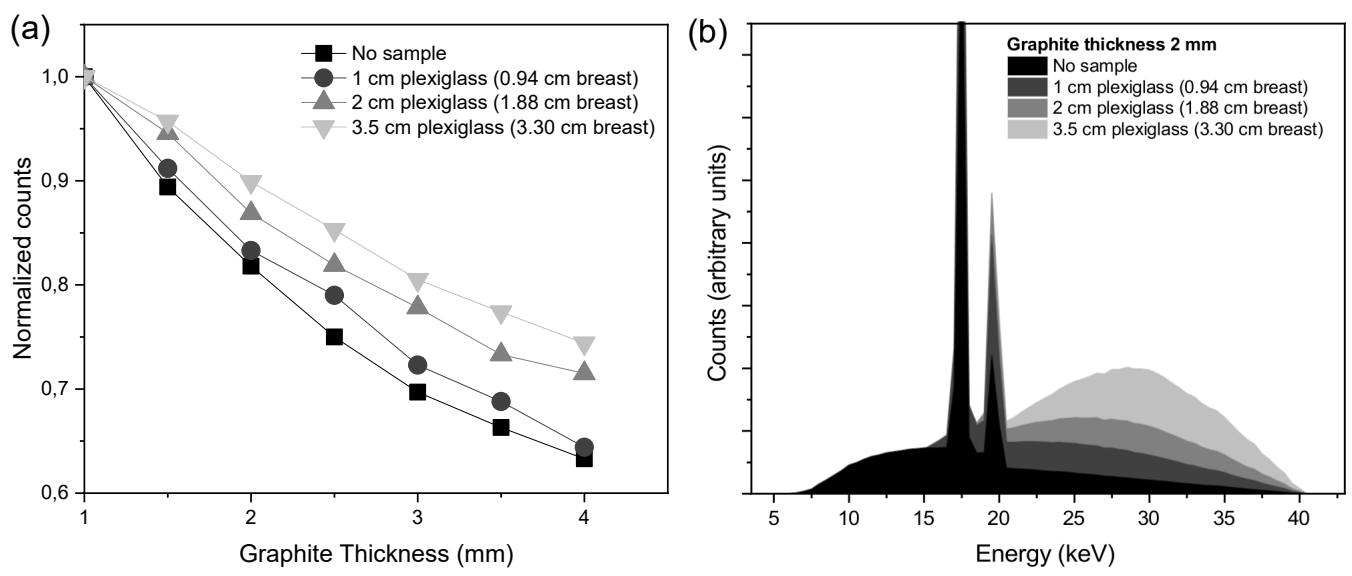

Figure 5. Panel (a) shows the attenuation of the beam as function of different graphite thickness and sample size. Each curve is normalized to the maximum and starting point is $1 \mathrm{~mm}$ corresponding at the setup currently used with about 0.5 $\mathrm{mm}$ graphite substrate for each mask. Panel (b) shows the beam hardening using $2 \mathrm{~mm}$ of graphite for different breast thickness.

$\mathrm{keV})$ which is close to the one of breast tissue $\left(0.89 \mathrm{~cm}^{-1}\right)$. Each curve reported in Fig.3 is normalized to its maximum, showing the percentage of the beam absorbed by the graphite only. The percentage of absorbed beam for any graphite thickness decreases with sample size. This is because graphite absorbs mostly the low energy part of X-ray spectrum, which is already significantly removed by the sample as its thickness increases, as also shown by the spectra shown in Fig.3b for different breast thickness and constant graphite thickness of $2 \mathrm{~mm}$. It should be observed that, in the absence of a sample, the transmission through an overall graphite thickness of $2 \mathrm{~mm}$ is reduced by $20 \%$. This requires an increase of the integration time of a factor 1.2 to match the same signal to noise ratio performances as the current laboratory scanner. 


\section{RESULTS}

The performance of the laboratory EI phase contrast scanner has been evaluated using ethically approved breast tissue specimens from the Barts tissue bank (St. Bartholomew's Hospital, London, UK). Samples have a radius of about $3 \mathrm{~cm}$ fitting the FoV of our scanner and have been selected by an experienced pathologist in order to ensure that a reasonable number of samples contain tumour margins. They are chemically fixed by paraformaldehyde immersion for $24 \mathrm{~h}$. For each sample, different images are acquired while exploiting all operating modes available for our phase contrast scanner, including radiography and tomography. Single image phase retrieval has been used for all data resulting in CT scans. ${ }^{30}$ Examples of the results achievable with the proposed system on breast samples are shown in Fig.6.
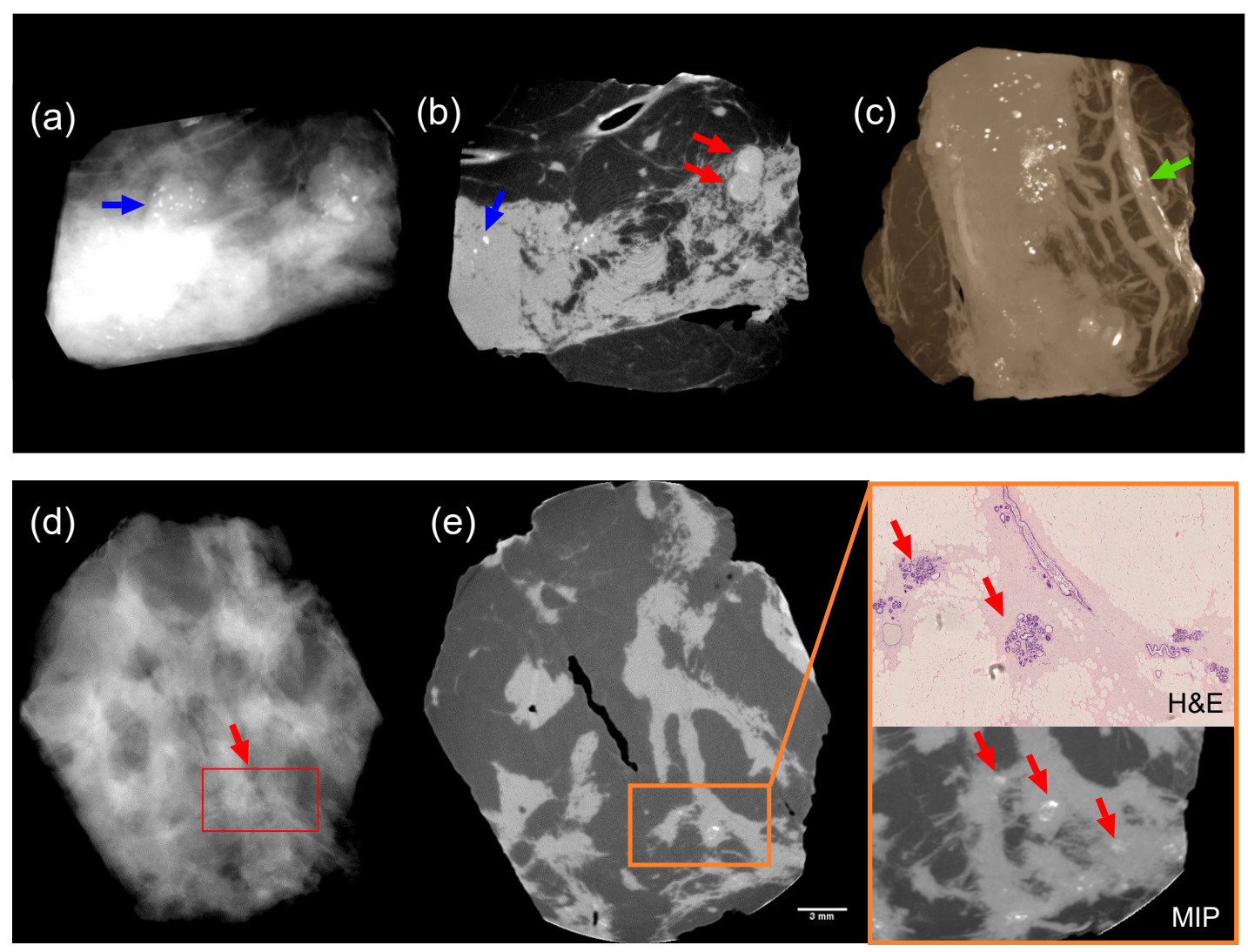

Figure 6. Panel (a) to (c) show 2D phase contrast radiography acquired with our EI system on a sample including a portion of malignant lesion, a single tomography slice and a 3D rendering of the same sample. Small calcifications are highlighted by blue arrows. Red arrows point at some faint calcified globular features while the green arrow highlights a duct covered by calcifications. Panel (d) and (e) show 2D phase contrast radiography of healthy sample and a tomography slice of the same sample, respectively. The inset reports a magnified view of an HE histological section and the maximum intensity projection (MIP) of the same region. Small calcifications in lobules are easily seen (red arrows). Red box in 2D phase contrast radiography in panel (d) highlights the same region where calcifications are detected in the tomographic slice.

2D phase contrast radiography is acquired within few seconds (see Fig.6a). This 2D mode would allow the surgeon to look at a given specimen from a series of orientations in less than one minute. In addition to the standard attenuation signal, refraction increases visibility of fine details providing a better view on fine spiculated structures. ${ }^{34}$ Detection of spiculations is a key factor in the assessment of the malignant nature of a breast mass. Thus, an improved visibility will positively impact diagnosis. In a subset of cases, phase contrast radiography could be sufficient to determine whether further tissue excision is required or not, without having to rely on the longer tomographic scan. When 2D XPCi does not provide sufficiently reliable information about the excisions 
margins, the phase contrast tomography mode would be used, providing an extra, powerful mechanism to access additional detail visibility. A tomographic slice obtained with the presented scanner is shown in Fig.6b. While the presence of calcifications can be appreciated in both radiographic and tomographic images (see blue arrows in Fig.6a and b), the tomographic slice clearly shows many additional features, such as some calcified, globular structures (red arrow in Fig.6b) compatible with a malignant feature, almost invisible in the 2D image due to tissue overlap. In addition, depending on the surgeons preference, the whole specimen volume could be shown on a semi-transparent 3D rendering, instead of having to scroll the volume slice by slice, as shown in Fig.6c. The $3 \mathrm{D}$ view provides better evidence of an enlarged duct (see green arrow in Fig.6c) being fitted with calcifications, which is hardly detected in the 2D phase contrast radiography. As an additional means to highlight features of interest, 3D information obtained from tomography can be reduced to $2 \mathrm{D}$ by the maximum intensity projection approach, as shown in Fig.6e. This mode provides a visualization tool closer to the current medical practice, but with an increased amount of information that is not achievable with standard 2D imaging, as clearly seen by comparing a maximum intensity projection (see Fig.6e) to a 2D projection (see Fig.6d). In the reported example, XPCi maximum intensity projection shows small calcifications invisible in the standard 2D image, as seen in the region highlighted by the red box and arrows in the phase contrast radiography (see Fig.6d) and in maximum intensity projection (see Fig.6e), respectively. HE staining in the inset confirms the presence of calcifications, and in addition it reveals their location in lobules. The increase in visibility of calcifications is an important achievement, since these features can often be pre-cancerous lesions. Preventing tissue overlap, XPC tomography delivers images that can be morphologically compared directly to histology, thus having a high diagnostic value in surgery as well as in histopathology.

\section{CONCLUSIONS}

This work demonstrates that a compact phase contrast tomography scanner based on edge illumination can be designed and scaled up to cover the imaging needs of the current breast surgery practice. The preliminary results obtained on breast samples reveals the high versatility of the designed machine delivering valuable diagnostic information not achievable with current imaging systems available in hospitals. The scanner can be used as a 2D radiography machine obtaining high quality phase contrast radiography in a time similar to instruments currently available in hospitals. In addition, a tomography imaging mode is available for the cases where 2D XPCI does not provide sufficient information. The simultaneous availability of $2 \mathrm{D}$ and volumetric imaging combined with the boost in fine details and contrast between soft tissues provided by phase contrast delivers images with outstanding potential benefits in conservative breast surgery, with the potential to reduce the number of reoperations and therefore both patient stress and costs for the healthcare service.

\section{ACKNOWLEDGMENTS}

This work was supported by the Wellcome Trust [200137/Z/15/Z]. M.E. was supported by the Royal Academy of Engineering under the RAEng Research Fellowships scheme.

\section{REFERENCES}

[1] Massimi, L., Bukreeva, I., Santamaria, G., Fratini, M., Corbelli, A., Brun, F., Fumagalli, S., Maugeri, L., Pacureanu, A., Cloetens, P., et al., "Exploring alzheimer's disease mouse brain through x-ray phase contrast tomography: From the cell to the organ," NeuroImage 184, 490-495 (2019).

[2] Cedola, A., Bravin, A., Bukreeva, I., Fratini, M., Pacureanu, A., Mittone, A., Massimi, L., Cloetens, P., Coan, P., Campi, G., et al., "X-ray phase contrast tomography reveals early vascular alterations and neuronal loss in a multiple sclerosis model," Scientific Reports 7(1), 5890 (2017).

[3] Bukreeva, I., Campi, G., Fratini, M., Spanò, R., Bucci, D., Battaglia, G., Giove, F., Bravin, A., Uccelli, A., Venturi, C., et al., "Quantitative 3d investigation of neuronal network in mouse spinal cord model," Scientific reports 7, 41054 (2017).

[4] Massimi, L., Fratini, M., Bukreeva, I., Brun, F., Mittone, A., Campi, G., Spanò, R., Mastrogiacomo, M., de Rosbo, N. K., Bravin, A., et al., "Characterization of mouse spinal cord vascular network by means of synchrotron radiation x-ray phase contrast tomography," Physica Medica 32(12), 1779-1784 (2016). 
[5] Mittone, A., Ivanishko, Y., Kovalev, S., Lisutina, P., Lotoshnikov, M., Tkachev, S., Tkacheva, M., Crippa, L., Dmitriev, V., and Bravin, A., "High resolution hard x-ray 3d mapping of a macaca fascicularis eye: A feasibility study without contrast agents," Physica Medica 51, 7-12 (2018).

[6] Vittoria, F. A., Kallon, G. K., Basta, D., Diemoz, P. C., Robinson, I. K., Olivo, A., and Endrizzi, M., "Beam tracking approach for single-shot retrieval of absorption, refraction, and dark-field signals with laboratory x-ray sources," Applied Physics Letters 106(22), 224102 (2015).

[7] Olivo, A. and Speller, R., "A coded-aperture technique allowing x-ray phase contrast imaging with conventional sources," Applied Physics Letters 91(7), 074106 (2007).

[8] Pfeiffer, F., Weitkamp, T., Bunk, O., and David, C., "Phase retrieval and differential phase-contrast imaging with low-brilliance x-ray sources," Nature physics 2(4), 258 (2006).

[9] Endrizzi, M., Vittoria, F. A., Diemoz, P. C., Lorenzo, R., Speller, R. D., Wagner, U. H., Rau, C., Robinson, I. K., and Olivo, A., "Phase-contrast microscopy at high x-ray energy with a laboratory setup," Optics letters 39(11), 3332-3335 (2014).

[10] Töpperwien, M., Krenkel, M., Vincenz, D., Stöber, F., Oelschlegel, A. M., Goldschmidt, J., and Salditt, T., "Three-dimensional mouse brain cytoarchitecture revealed by laboratory-based x-ray phase-contrast tomography," Scientific reports 7, 42847 (2017).

[11] Bravin, A., Coan, P., and Suortti, P., "X-ray phase-contrast imaging: from pre-clinical applications towards clinics," Physics in Medicine \& Biology 58(1), R1 (2012).

[12] Castelli, E., Tonutti, M., Arfelli, F., Longo, R., Quaia, E., Rigon, L., Sanabor, D., Zanconati, F., Dreossi, D., Abrami, A., et al., "Mammography with synchrotron radiation: first clinical experience with phase-detection technique," Radiology 259(3), 684-694 (2011).

[13] Schültke, E., Fiedler, S., Nemoz, C., Ogieglo, L., Kelly, M. E., Crawford, P., Esteve, F., Brochard, T., Renier, M., Requardt, H., et al., "Synchrotron-based intra-venous k-edge digital subtraction angiography in a pig model: A feasibility study," European journal of radiology 73(3), 677-681 (2010).

[14] Renier, M., Brochard, T., Nemoz, C., Requardt, H., Bräuer, E., Esteve, F., Balosso, J., Suortti, P., Baruchel, J., Elleaume, H., et al., "The radiotherapy clinical trials projects at the esrf: technical aspects," European journal of radiology $\mathbf{6 8}(3)$, S147-S150 (2008).

[15] Lewis, R., "Medical phase contrast x-ray imaging: current status and future prospects," Physics in medicine $\mathscr{G}$ biology 49(16), 3573 (2004).

[16] Cabioglu, N., Hunt, K. K., Sahin, A. A., Kuerer, H. M., Babiera, G. V., Singletary, S. E., Whitman, G. J., Ross, M. I., Ames, F. C., Feig, B. W., et al., "Role for intraoperative margin assessment in patients undergoing breast-conserving surgery," Annals of surgical oncology 14(4), 1458-1471 (2007).

[17] Fleming, F., Hill, A., Mc Dermott, E., O'Doherty, A., O'Higgins, N., and Quinn, C., "Intraoperative margin assessment and re-excision rate in breast conserving surgery," European Journal of Surgical Oncology (EJSO) 30(3), 233-237 (2004).

[18] Veronesi, U., Luini, A., Galimberti, V., and Zurrida, S., "Conservation approaches for the management of stage i/ii carcinoma of the breast: Milan cancer institute trials," World journal of surgery 18(1), 70-75 (1994).

[19] Endrizzi, M., Vittoria, F., Rigon, L., Dreossi, D., Iacoviello, F., Shearing, P., and Olivo, A., "X-ray phasecontrast radiography and tomography with a multiaperture analyzer," Physical review letters 118(24), 243902 (2017).

[20] Endrizzi, M., Vittoria, F. A., Kallon, G., Basta, D., Diemoz, P. C., Vincenzi, A., Delogu, P., Bellazzini, R., and Olivo, A., "Achromatic approach to phase-based multi-modal imaging with conventional x-ray sources," Optics Express 23(12), 16473-16480 (2015).

[21] Kallon, G. K., Wesolowski, M., Vittoria, F. A., Endrizzi, M., Basta, D., Millard, T. P., Diemoz, P. C., and Olivo, A., "A laboratory based edge-illumination x-ray phase-contrast imaging setup with two-directional sensitivity," Applied Physics Letters 107(20), 204105 (2015).

[22] Modregger, P., Cremona, T. P., Benarafa, C., Schittny, J. C., Olivo, A., and Endrizzi, M., "Small angle x-ray scattering with edge-illumination," Scientific reports 6, 30940 (2016).

[23] Malecki, A., Potdevin, G., and Pfeiffer, F., "Quantitative wave-optical numerical analysis of the dark-field signal in grating-based x-ray interferometry," EPL (Europhysics Letters) 99(4), 48001 (2012). 
[24] Rigon, L., Besch, H.-J., Arfelli, F., Menk, R.-H., Heitner, G., and Plothow-Besch, H., "A new dei algorithm capable of investigating sub-pixel structures," Journal of Physics D: Applied Physics 36(10A), A107 (2003).

[25] Endrizzi, M. and Olivo, A., "Absorption, refraction and scattering retrieval with an edge-illumination-based imaging setup," Journal of Physics D: Applied Physics 47(50), 505102 (2014).

[26] Hagen, C., Diemoz, P., Endrizzi, M., Rigon, L., Dreossi, D., Arfelli, F., Lopez, F., Longo, R., and Olivo, A., "Theory and preliminary experimental verification of quantitative edge illumination x-ray phase contrast tomography," Optics express 22(7), 7989-8000 (2014).

[27] Zamir, A., Hagen, C., Diemoz, P. C., Endrizzi, M., Vittoria, F., Chen, Y., Anastasio, M. A., and Olivo, A., "Recent advances in edge illumination x-ray phase-contrast tomography," Journal of Medical Imaging 4(4), 040901 (2017).

[28] Munro, P. R. and Olivo, A., "X-ray phase-contrast imaging with polychromatic sources and the concept of effective energy," Physical Review A 87(5), 053838 (2013).

[29] Hagen, C. K., Endrizzi, M., Diemoz, P. C., and Olivo, A., "Reverse projection retrieval in edge illumination x-ray phase contrast computed tomography," Journal of Physics D: Applied Physics 49(25), 255501 (2016).

[30] Diemoz, P., Hagen, C., Endrizzi, M., Minuti, M., Bellazzini, R., Urbani, L., De Coppi, P., and Olivo, A., "Single-shot x-ray phase-contrast computed tomography with nonmicrofocal laboratory sources," Physical Review Applied 7(4), 044029 (2017).

[31] Reichenbach, S. E., Park, S. K., and Narayanswamy, R., "Characterizing digital image acquisition devices," Optical Engineering 30(2), 170-178 (1991).

[32] Diemoz, P. C., Vittoria, F. A., and Olivo, A., "Spatial resolution of edge illumination x-ray phase-contrast imaging," Optics express 22(13), 15514-15529 (2014).

[33] Ignatyev, K., Munro, P., Speller, R., and Olivo, A., "Effects of signal diffusion on x-ray phase contrast images," Review of Scientific Instruments 82(7), 073702 (2011).

[34] Pisano, E. D., Johnston, R. E., Chapman, D., Geradts, J., Iacocca, M. V., Livasy, C. A., Washburn, D. B., Sayers, D. E., Zhong, Z., Kiss, M. Z., et al., "Human breast cancer specimens: diffraction-enhanced imaging with histologic correlationimproved conspicuity of lesion detail compared with digital radiography," Radiology 214(3), 895-901 (2000). 